perception of the passage of time and then by hallucinations There is also an idiosyncratic reaction resembling a psychosis; this is not dose-dependent, and the probability of its occurrence is apparently not related to the stability of the personality. Rarely hallucinations and fragmentation of thought processes occur and present as psychotic disturbances. The physical signs may include variations in blood pressure and pulse rate, bronchoconstriction, hypoglycaemia, and alimentary disturbances, though these features are not constant. More significant is the marked dilatation of conjunctival blood vessels which frequently occurs after first taking the drug.

The World Health Organization has defined a state of drug dependence of the cannabis type. ${ }^{5}$ There is a moderate to strong psychological dependence as a result of a desire for the subjective effects, but an absence of physical dependence-so no abstinence syndrome occurs-and there is no evidence of tolerance. Commenting on this, N. B. Eddy and his colleagues stated that individuals dependent on cannabis might suffer from " inertia, lethargy, self neglect, feelings of increased capability with consequent failure, and precipitation of psychotic episodes."

Research into the effects of cannabis in man is in progress in the U.S.A. and elsewhere. ${ }^{6}$ No objective assessment of the effects of prolonged administration of the drug has yet been published. There is still disagreement whether or not cannabis users are likely to progress to heroin and other " hard" drugs. An answer to both these questions is urgent. But while the effects of prolonged exposure to cannabis remain speculative it is irresponsible to encourage a more permissive attitude to the drug.

\section{Respiratory Burns}

On 28 November 1942 a disastrous fire occurred at the Cocoanut Grove night club in Boston in which 491 people lost their lives. Of the 114 casualties taken to the Massachusetts General Hospital, 75 were either dead on arrival or died of anoxia within minutes. Of the 39 who survived long enough to be treated, only three were entirely without respiratory symptoms. The clinical picture, ${ }^{1} x$-ray appearances, ${ }^{2}$ and pathology ${ }^{3}$ of these pulmonary injuries were well recorded. Only a small proportion of the victims had extensive burns, and most of the deaths were attributed to anoxaemia and poisoning by carbon monoxide and traces of oxides of nitrogen.

In 1945 A. R. Moritz ${ }^{4}$ and his co-workers published experimental work on dogs in which they showed that when hot air was blown into the larynx at $270^{\circ} \mathrm{C}$. the temperature of the air fell to $50^{\circ} \mathrm{C}$. in the trachea. Hot air and flame could burn the larynx and trachea but failed to produce pulmonary damage. On the other hand, inhaled steam produced severe pulmonary injury owing to its greater latent heat.

A review by $A$. W. Phillips and colleagues of cases treated at the Massachusetts General Hospital during $1939-57^{5-7}$ showed that in $42 \%$ of fatal cases the patient died of damage to the respiratory tract, about half of these in the first three days. They stressed the significance of deep flame burns around the nose and mouth, burning in an enclosed environment, and the inhalation of smoke and the products of incomplete combustion as ominous warning signs of pulmonary damage even in the absence of respiratory signs and symptoms.

Recently H. H. Stone and his colleagues, ${ }^{8}$ dissatisfied with the results of treating patients with pulmonary burns (mortality $89 \%$ ), carried out experiments to evaluate present methods of therapy. They insufflated steam at $10 \mathrm{~mm}$. $\mathrm{Hg}$ pressure through a chilled, plastic, intratracheal tube for one to five second in rats. Their studies showed that treatment with $40 \%$ oxygen or $80-100 \%$ humidity reduced the mortality of the animals. Antibiotics failed to decrease mortality, and treatment with adrenal steroids appeared to be contraindicated.

Respiratory tract damage in burned patients is less common in Britain than in the United States. In Britain extensive burns affecting the face are usually the result of clothes being set alight from unguarded coal fires, and not from people being trapped in burning buildings. The victims, usually children, run about breathing normal air till the flames are put out. In this country only about $5 \%$ of patients admitted to hospital with domestic burns have received them in conflagrations, and a pulmonary lesion, because it is uncommon, tends to be missed until a disaster like the Dellwood Maternity Home fire, ${ }^{9}$ which killed 13 newborn babies, reminds us of the truism that "smoke is poison."

Clinically, the surgeon should suspect damage of the pulmonary tract from the circumstances in which the patient was burned. He must then assess the site of the damage. If the pharynx, larynx, and upper trachea alone are injured the patient will probably complain of a sore throat and hoarseness-possibly leading to stridor. He may also have a cough, with carbon particles in the sputum and perhaps flecks of blood. If pulmonary damage is present it may not be apparent for several hours, or it may kill the patient with anoxia in a matter of minutes.

Stone's classification of pulmonary damage into respiratory insufficiency, pulmonary oedema, and pulmonary infection is a practical one ; the first two stages may not be clinically obvious in a mild case.

Pulmonary insufficiency may last 24-36 hours. It is the result of narrowing of the smaller bronchi due to spasm, congestion, and oedema of the walls, and blocking with carbon particles, epithelium, fibrin, leucocytes, and mucus. This leads to laboured breathing with an expiratory wheeze. Anoxia is evident from restlessness and confusion, and it may lead to coma and permanent brain damage. Cyanosis is usual, but it may be masked by anaemia or the cherry-red of carbon monoxide poisoning. If rales are present within an hour the prognosis is grave, but even if they are absent the victim should be admitted to hospital overnight for observation like a case of concussion. A single normal chest radiograph is of no significance. Changing physical signs in the chest are probably due to transient atelectasis and filling of alveoli with exudate, epithelial debris, and white cells. Stone's work has shown the value of treating burned patients in this stage with $40 \%$ oxygen and $80-100 \%$ humidity. Positive-pressure ventilation did not appear to overcome hypoxia, and tracheostomy, except for associated laryngeal obstruction, may precipitate pulmonary oedema by reducing the intrapulmonary pressure produced by intermittent glottic obstruc-

1 Aub, J. C., Pittman, H., and Brues, A. M., Ann. Surg., 1943, 117, 834, Schatzki, R., ibid., 1943, 117, 841.

Schatzki, R., ibid., 1943, 117, 841. J., ibid., 1943, 117, 865.

"Mallory, T. B., and Brickley, W. J., ibid., 1943, 117, 865. $1945,21,311$.

5 Phillips, A. W., and Cope, O., Ann. Surg., 1962, 155, 1.

- 1 ibid., 1962, 156, 759.

' - Tanner, J. W., and Cope, O., ibid., 1963, 158, 799.

Stone, H. H., Rhame, D. W. Corbitt, J. D., Given, K. S., and Marün, J. D., ibid., 1967, 165, 157 .

Cox, M. E., Heslop, B. F., Kempton, J. J., and Ratclif, R. A., Brit. med. F., 1955, 1, 942. 
tion to respiration. Aminophylline may help to relieve bronchospasm.

Pulmonary oedema, which is uncommon within eight hours of the burn, usually follows resuscitation. Strictly aseptic positive-pressure ventilation is the only useful therapy ${ }^{8}$ and may be given through a tracheostomy, cuffed tube, or facepiece. Pulmonary infection is the final condition through which all cases of pulmonary damage must pass, and it calls for antibiotics in high dosage controlled by daily cultures.

First aid for scalds has been in the public eye in recent months, particularly in the controversial matter of immediate cooling of the scalded skin. But there is no controversy about the value of a first-aid precaution against smoke and toxic fumes. The three survivors of the Cocoanut Grove disaster who sustained no damage to the respiratory tract had covered their mouths with wet clothes. Is this not a measure which television playwrights could teach by portrayal without comment?

\section{Sjögren's Syndrome}

If a woman in her 40s complains of dry, smarting, burning, or itching eyes and a dry mouth, then the possibility of Sjögren's syndrome ${ }^{1}$ should be considered. The other common component of the syndrome is rheumatoid arthritis, but this may be replaced or overshadowed by enlargement of the parotid or lacrimal gland, purpura, Raynaud's phenomenon, subcutaneous nodules, dry skin, scleroderma, splenomegaly, or peripheral lymphadenopathy. ${ }^{2}$ Thus the patient may be seen in either the ophthalmic or the rheumatology clinic, thereby determining which set of investigations will be undertaken. The ophthalmologist will insert Schirmer filter paper inside the lower eyelid near the external canthus to demonstrate deficiency of lacrimal secretion; a drop of rose-bengal dye to demonstrate obvious staining of the bulbar conjunctiva and cornea ; and he may find, by slit-lamp examination, punctate corneal staining with fluorescein. By these means the presence of keraconjunctivitis sicca may be confirmed. The rheumatologist, on the other hand, will seek confirmation of the clinical diagnosis of rheumatoid arthritis by radiological and serological examinations. It is particularly the field of serology which has contributed richly to our knowledge of the disorder and extended the perspectives of its aetiology and pathogenesis. B. R. Jones ${ }^{3}$ found that the sera of patients with Sjögren's syndrome contained specific precipitating antibodies reacting with extracts of salivary and lacrimal glands, while $J$. R. Anderson and his colleagues ${ }^{4}$ detected non-organ-specific precipitating autoantibodies reacting with a variety of extracts of human and some animal tissues and thyroglobulin. Serological tests suggestive of systemic lupus erythematosus are also a feature of Siögren's syndrome. J. M. Heaton ${ }^{5}$ demonstrated the frequent occurrence of the lupus-erythematosus cell test, subsequently showing with $\mathrm{D}$. Doniach that the antinuclear factor and thyroid autoantibodies occurred in about a half of patients with Sjögren's syndrome. The rheumatoid factor was found in $82 \%$ of cases ; it was present in the same proportion whether or not there was joint disease. ${ }^{6}$ In a still wider clinicoserological survey in Bethesda in the United States evidence was found not only of distinct clinical but also of serological subgroups. ${ }^{7}$ The presence of rheumatoid factors in almost every case of Sjögren's syndrome, even in the absence of rheumatoid arthritis, was an unexpected finding.

All the evidence points to the fact that Sjögren's disease is an autoimmune phenomenon, and the pathological findings support this view. Thus lacrimal and salivary glands are infiltrated by lymphocytes and plasma cells, with atrophy of the secreting acini and increased interstitial fibrosis. Similar infiltrative changes are widespread, as might be expected in a disorder which has a chameleon-like variety of clinical masquerades. Moreover, though the syndrome may present and be investigated in ophthalmic and rheumatology clinics, it may be found by diligent search in patients seen in psychiatric and gastroenterology clinics. Sjögren's syndrome is predominantly a disorder of menopausal women, many of whom are also suffering from endogenous or reactive depression. It may occur simultaneously with another autoimmune process-primary biliary cirrhosis-which is also common in women in their 40s. Under these circumstances depression and primary biliary cirrhosis may prove dominant and mask concomitant Sjögren's disease unless the serological abnormalities of the latter are specifically sought.

It remains a distressing affliction since treatment is so unsatisfactory. Local treatment to the eyes for relief of symptoms due to dryness includes $1 \%$ methylcellulose in saline (artificial tears) or sulphacetamide eye drops. The latter are bland and alkaline and minimize bacterial complications. The danger of corneal ulceration is always present, so that corticosteroid drops should incorporate an antibiotic. Sealing of the puncta by cautery helps to conserve what little moisture is present, and contact lenses may give relief if these are tolerated. Because of its similarities to rheumatoid arthritis and systemic lupus erythmatosus, J. M. Heaton ${ }^{8}$ conducted a double-blind trial comparing the value of giving $800 \mathrm{mg}$. hydroxychloroquine daily for six months with a placebo. A significant difference was obtained, for 12 of 15 improved with hydroxychloroquine compared with 6 of 15 while on the placebo. Two patients developed toxicity owing to deposition of the drug in the cornea, which took two months to appear and about five months to disappear after the sixmonth course of hydroxychloroquine had ended. Antimalarial drugs are particularly indicated if an appreciable degree of rheumatoid arthritis is also present. Because of its putative autoimmune nature the syndrome has also been treated by immunosuppressive drugs. Thus corticosteroid drugs have been used both locally and by mouth, but relapses have followed cessation of treatment and the danger of corneal ulceration is a real one. Since it commonly occurs in menopausal women, oestrogens might be found to be helpful ; and this association is strengthened by the fact that excretion of hydroxyproline, which is greatly raised in Sjögren's syndrome, may be corrected by oestrogen administration. If the depression is endogenous rather than due to the distressing affliction itself, then a therapeutic trial of nortriptyline or imipramine is worthwhile.

1 Sjögren, H., Acta ophthal. (Kbh.), 1933, Suppl. No. 2.

- Vanselow, N. A., Dodson, V. N., Angell, D. C., and Duff, I. F., Ann intern. Med., 1963, 58, 124.

s Jones, B. R., Lancet, 1958, 2, 773.

- Anderson, J. R., Gray, K. G., Beck, J. S., and Kinnear, W. F., ibid. $1961,2,456$.

- Heaton, J. M., Brit. med. f., 1959, 1, 466.

- Proc. roy. Soc. Med., 1962, 55, 479.

' National Institutes of Health Staff Conference, Ann. intern. Med., 1964, 61, 509.

- Heaton, J. M., Amer. f. Ophthal., 1963, 55, 983. 